\title{
Research and Practice on Data Governance of Aerospace High-Tech Enterprises
}

\author{
Xinjian Liang, Lin Guo, Long Zhao \\ Beijing Institute of Aerospace Control Devices, Beijing, China
}

Email address:

eryazi0425@sina.com (Xinjian Liang), zhaobohui@163.com (Lin Guo), 15611796238@163.com (Long Zhao)

\section{To cite this article:}

Xinjian Liang, Lin Guo, Long Zhao. Research and Practice on Data Governance of Aerospace High-Tech Enterprises. Science Discovery. Vol. 6, No. 1, 2018, pp. 57-61. doi: 10.11648/j.sd.20180601.20

Received: January 16, 2018; Accepted: January 31, 2018; Published: May 23, 2018

\begin{abstract}
With increasing demands on refinement, datamation and standardization of the aerospace high-tech enterprises businesses and the accelerating pace of the informatization construction, data governance has became an important foundation for the development of aerospace high-tech products, improvement of products quality, and intelligence analysis and decision. At present, the potential value of the data assets of aerospace high-tech enterprises have not yet been excavated, data governance still lays emphasis on technologies, lack of top-level planning for data governance, the effective data governance mechanism not yet been established, lack of coordination and cooperation between different business departments. Although every business department has collected and displayed of the core businesses and products data, but lack of the deeper enterprise-level data governance system. The data governance is a complex system engineering, which should be promoted to the core strategy of the enterprise. The data governance system of aerospace high-tech enterprises is made up of an integrated and balanced informatization architecture involved in organization, data, technology and application. Through the research and practice on the master data management,unifying data coding rules, collecting data, sharing data, data life cycle management and data application, meeting the requirement of the aerospace high-tech enterprises and promoting the data to bacome the largest value source.
\end{abstract}

Keywords: Data Governance, Big Data, Master Data Management, Data Architecture, Intelligence Analysis and Decision

\section{航天高技术企业数据治理研究与实践}

梁新建, 郭琳, 赵龙

北京航天控制仪器研究所, 北京, 中国

邮箱

eryazi0425@sina.com (梁新建), zhaobohui@163.com（郭琳），15611796238@163.com（赵龙）

摘要：随着航天高技术企业业务精细化、数据化、标准化的要求越来越高和信息化建设步伐不短加快, 数据治理已成 为航天高技术产品研制、提升产品质量、智能分析决策的重要基础。目前, 航天高技术企业数据资产的价值和潜力还 远远没有被挖掘出来, 数据治理工作仍然偏重于技术, 缺乏顶层规划, 数据治理机制尚未建立, 缺乏业务部门之间的 相互协调与配合。数据治理工作主要还停留在核心业务及产品的数据收集与展示等方面, 而对企业级更深层次的数据 治理体系建设尚未涉及。数据治理是一项复杂而艰巨的系统工程，应该提升到企业的核心战略层面。本文通过航天高 技术企业数据治理研究, 建立了均衡的包括组织、数据、技术与应用的数据治理体系架构。通过对主数据管理、数据 编码规则、数据收集、数据共享、数据全生命周期管理与数据深度应用等方面的研究与实践, 满足航天高技术企业数 据使用需求，逐步推动数据成为航天高技术企业最大的价值来源。 
关键词: 数据治理, 大数据, 主数据管理, 数据架构, 智能分析决策

\section{1. 引言}

马云说: 未来的世界, 我们将不再由石油驱动, 而是 由数据驱动。随着世界从IT (Information Technology) 时 代向DT（Data Technology）时代进化, 数据的重要性大 大增强, 数据已经成为现代企业最大的价值来源, 是支持 企业发展的重要推动力。

目前, 大多数航天高技术企业为了提升航天产品质量 和企业治理科学性, 已经开始重视数据治理工作, 通过引 入数据治理工具和理念 [1], 加快推进商业智能、领导驾驶 舱、产品数据包、主数据管理等系统建设工作, 有力提升 了航天高技术企业的科学治理、精益管理能力[2], 并取得 了初步效果。

但是, 航天高技术企业的数据治理工作尚处于处级阶 段, 大多数航天企业的数据资产的价值和潜力还远远没有 被挖掘出来, 多年积累数据的应用和价值都大打折扣, 其 中最重要的原因是这些企业缺乏完整的数据治理机制。如 何系统的对数据进行治理已经成为困扰航天高技术企业 的一项巨大挑战, 数据治理是一项复杂而艰巨的系统工程, 并不仅仅是信息化部门的职责, 需要提升到企业的核心战 略层面给予关注。

\section{2. 航天高技术企业数据治理的现状和问题}

如今, 全球各类组织都认识到数据资产的重要性和价 值[3]。与此同时, 大家发现自己并未充分利用这些数据资 产，原因在于企业中的大量数据缺乏准确性、一致性、相 关性和及时性[4]。航天高技术企业的数据治理已经开始起 步，但面临着各方面的问题。

\section{1. 航天高技术企业数据治理还处于初级阶段}

航天高技术企业在数据管理组织方面存在问题, 大多 数企业缺乏统一归口的数据管理组织, 存在数据管理职责 分散、能力不足、权责不明的问题，可谓“人人管理、无 人负责”。主要原因是缺乏数据规范、缺乏数据管理监督 措施、缺乏数据管理考核, 无法保证数据管理的有效执行 [5]。

另外, 在数据标准方面, 各业务部门制定自己的业务 标准, 只关心自己的业务数据, 数据共享能力差。尚未建 立企业级的数据编码, 各类数据编码规则不一致, 甚至发 生冲突。

在数据质量方面, 目前大多数航天企业的数据管理主 要由各部门分头进行, 面临管理人员不足、经验不够、监 管方式不全面等问题。数据审核停留在部门内部, 部门间 缺乏交流, 各自为政。导致跨专业数据沟通机制不完善, 缺乏数据质量管控规范和标准, 缺乏完善的数据质量管控 流程和系统支撑能力, 降低了数据质量。
在数据安全方面, 目前航天企业对数据安全的认识主 要集中在数据安全策略和用户访问控制等方面, 在数据安 全事件处理和数据风险管理等方面认识需要提升。

在数据全生命周期管理方面, 航天企业缺乏完善和统 一的从数据产生、使用、维护、备份到销毁的数据全生命 周期管理规范和流程, 不能确定过期和无效数据的识别条 件, 且非结构化数据未被纳入数据管理范畴。另外, 信息 化工具支撑数据状态查询能力不足。

总之, 航天高技术企业的数据就像一个待开采的含有 丰富矿藏的矿山, 而数据治理工作还处于初级阶段。

\section{2. 航天高技术企业对数据治理的认知存在差距}

航天高技术企业对数据治理的认知主要包括业务人 员对数据收集、存储、分析、挖掘和可视化的认知。目前, 航天高技术企业主要集中收集结构化数据, 对于实时数据 的收集程度较低, 而这些实时数据恰恰反映了企业发展过 程中的动态性、实时性和全面性, 是企业实现科学管理的 重要数据支撑, 数据不全面将影响决策的科学性。航天高 技术企业应重视对非结构数据、实时数据的收集, 只有建 立了全面、系统的数据仓库, 并加强数据治理和应用, 才 能发挥数据的价值。

\section{3. 数据治理在企业治理中还未发挥有效作用}

数据治理, 更重要在于应用引领, 而不单纯是技术问 题。数据治理作用的彰显是企业选择数据治理的直接推动 力[6]。其中, 企业要素的数据化、规范化是企业治理走向 决策精准化的基础。企业资源的快速组合，将满足企业业 务转型、业务拓展的不同需求, 提高企业治理的精准度。 管理流程的数据化将有力促进企业流程优化与业务重组, 降低运营成本。目前, 航天高技术企业并不完全清楚数据 治理的作用和功能, 盲目进行数据治理反而会增加运营成 本, 这成为企业数据治理的主要障碍。

\section{4. 航天高技术企业数据共享与开放程度不高}

航天高技术企业数据共享与开放程度不高, 随着企业 信息化的推进，“信息分散”和“信息孤岛”等新问题随之出 现, 这些问题出现影响了企业信息化的应用和推进。因此, 企业需要解决方案来处理这方面的问题, 来实现数据的有 序管理和共享。

\section{3. 航天高技术企业数据治理的总体思路}

航天高技术企业数据治理成效相对于应用系统、基础 设施建设、数据容灾备份等工作相对滞后。仅通过技术手 段、以及建系统的方式难以解决数据治理的所有问题。需 要通过数据治理体系建设形成可行方案。

要真正解决数据管理中的问题“功夫”在数据之外, 数 据治理的症结是管理机制问题。数据治理是要以实现数据 
标准化为目标, 以健全数据组织为保障, 以数据过程管控 为手段, 实现全面、高效的数据管理[7]。

\section{1. 数据治理工作目标}

数据治理工作目标包括三部分: 首先应建立数据管理 体系, 包括一套数据管理规范体系, 描述全所到底有哪些 数据及数据属性, 全企业说一种“语言”; 运行一套数据管 理职责体系, 描述各部门应承担的责任; 应用一套数据考 核评价体系, 描述数据管理的考核评价手段。

第二要建立数据管理工作机制, 把具体工作通过规范 的模板分解落实到具体部门, 督促其限时完成, 使数据管 理体系在实际工作中能够运转起来, 切实解决数据问题, 并将成果不断充实到数据管理体系中, 形成数据管理的良 性循环。

第三要构建数据管理软件平台, 即依据统一的数据标 准规范进行数据集中采集、加工、发布和应用, 并提供数 据监测和考核手段, 及时发现数据问题, 形成数据服务和 数据管控的一体化工作平台, 为数据管理体系提供有力的 系统支撑。

\section{2. 成立数据治理工作组, 强化组织保证}

要成立数据治理工作组, 强化组织机构在数据治理过 程的重要作用 $[8]$ 。在航天高技术企业顶层成立由高层管理 人员、信息管理部门和业务部门主要负责人组成的数据治 理领导小组。中间层成立数据治理工作组, 主要由各业务 部门业务专家、信息部门技术专家、数据库管理专家、变 更管理/培训等专家组成。最底层成立数据治理实施组, 主要由各管理信息系统项目组成员组成。

\section{3. 制定标准规范, 标准化工作先行}

目前, 航天高技术企业存在产品多品种、小批量的制 订化特点, 正在大力向产品化、通用化转变, 所以建立统 一的数据标准迫在眉睫。需要结合数据治理工作, 专门成 立数据标准工作组, 牵头组织完成各类标准制定, 主要包 括技术标准、业务标准、管理标准、数据质量标准等内容。 并结合业务需求、数据共享需求和数据挖掘需求建立数据 标准、数据标准管理、数据质量管理, 明确数据的定义, 提升数据规范化、标准化, 为数据质量提供质量保障。

\section{4. 强化数据治理体系建设, 形成长效机制}

航天高技术企业日常业务繁杂，技术攻关、研发创新 工作是中心工作, 目前员工已经处于加班加点的工作状态, 所以需要加强数据治理体系建设, 通过长效机制保证建设 效果。

数据治理的关键在于治理, 要作为一项专门的业务来 常态化地管理, 需要构建全流程、整周期、多方位、跨组 织、严考核的治理体系。要从数据产生的源头抓起, 覆盖 数据全生命周期各个环节。需要考虑到指标、共享、主数 据、元数据等数据架构的每个层次和细度。需要各业务单 元、业务部门、应用系统、基础设施、技术、建设、运维 等领域的全面参与和协调。通过明确建设目标和周期、细
化工作计划, 责任到人, 强化结果导向、严肃考核, 确保 体系运行效果。

\section{5. 结合航天企业特点, 推行自上而下的建设模式}

根据航天企业建设的实际情况及企业内对数据治理 的定位和出发点, 结合航天高技术企业信息化建设现状, 适合选择至上而下的建设模式开展数据治理体系建设, 并 将在主数据管理及应用、数据质量提升、数据安全管理强 化、信息生命周期管理、企业级数据架构设计与管理、全 方位数据应用等方面进行重点建设与关注。

\section{6. 建设数据管理软件平台, 确保数据治理效果}

在数据治理过程中, 需要通过数据技术对数据进行有 效控制与支撑，以元数据采集、管理和应用为核心，驱动 数据、标准、数据质量实现。另外, 充分利用航天企业在 产品数据管理方面的经验, 开展元数据管理平台、主数据 管理平台以及数据分析技术、数据质量整治技术的研究, 为了确保安全、国家保密要求以及建设效果, 航天企业数 据管理软件平台主要依据自身能力开展建设。

\section{4. 航天企业数据治理实践}

关于管理提升，大家最常挂在嘴边的一句话是 “三分 技术、七分管理、十二分数据”。在管理提升项目实施过 程, 系统实现和软件开发时间不超过项目实施周期的百分 之二十, 百分之八十以上的时间用在了贯穿全程的三大任 务上, 即培训、数据治理和管理变革, 其中尤以数据治理 工作量最大。

\section{1. 主数据管理在航天企业中的实践}

\subsection{1. 主数据定义}

主数据（Master Data，MD）定义为: 在企业中用来 定义业务对象的、具有持续性的、非交易类的数据。它在 整个企业内具有或应该具有一致的视图。常见的主数据类 型有产品、物料、供应商、会计科目、组织机构等。主数 据具有稳定性、核心性、相互关联性以及分类特性[8]。

主数据管理(Master Data Management, MDM)是基于 本企业特点的一组组数据管理最佳实践的集合 [9]。它融合 于业务应用系统、信息管理方法、数据管理工具之中。在 技术上支持对主数据的抽取、整合与分享利用，提供准确、 及时、一致、完整的主数据。

航天企业开展主数据管理项目, 不仅可以优化核心数 据, 提高数据质量, 而且可以带来显著的商业价值。一致、 准确、有效、完整的主数据可以为企业带来以下优势：降 低产品管理成本; 实现物资的统一采购; 通过准确的业务 数据提高决策效率; 提升市场分析能力与销售预测; 降低 多系统数据运维成本; 提高订单准确性, 降低物流成本。

\subsection{2. 主数据管理系统建设目标}

航天企业通过主数据管理系统的成功上线, 给企业进 一步推广主数据管理增加信心, 具体的实现目标包括: 
第一、建设主数据管理系统, 集成到企业的现有IT环 境中，合理利用现有的IT资源。通过防止在不同系统进行 多次处理, 降低总体数据维护成本。

第二、通过主数据类型不同制定合理的数据分发机制, 加速流程的执行。

第三、确保数据一致性和准确性, 降低有数据不一致 带来的错误处理成本。

第四、通过收集每个人提供的最新信息, 改善战略销 售和采购方面的策略。

第五、提供一套量身定制的IT和业务情景组合, 满足 具体客户在主数据管理方面的需求。

第六、提供一个模型驱动的平台方式, 拥有预配置的 对象模板。

第七、及时了解客户信息、掌握客户所需, 提高服务 质量、创造更大利润。

\subsection{3. 航天企业主数据管理系统建设及实践}

航天企业主数据管理系统建设具体包括了数据存储、 数据整合、数据清洗、数据发布、数据管理这五大功能。

第一、数据存储功能实现建立企业级、灵活可扩展的 数据模型、统一主数据资源库。

第二、数据整合功能是通过对多系统主数据合并, 将 数据进行批量导入, 实现对核心数据的集中管理。

第三、数据清洗功能实现对数据的补充、检验、查重、 排重等操作。

第四、数据发布功能是通过Web服务将统一、准确的 数据发送到所需的业务系统里。

第五、数据管理功能实现工作流的集成、变更管理、 版本管理、属性安全和日志管理。

\section{2. 制定编码规则, 建立编码体系}

提升数据质量过程中最难的是制定编码规则, 一个考 虑周全的编码体系需要跨部门反复讨论。不同的数据可能 有不同的编码结构, 但必须遵循共同的编码原则: 如唯一 性、简练性、规范性、便于系统处理、易用性等。另外, 编码问题绝不仅仅是一个技术问题, 要同时考虑管理现状、 业务特点、历史数据等方方面面。

北京航天控制仪器研究所完成了项目主数据的整理 工作。另外, 通过专题讨论, 北京航天控制仪器研究所制 定并发布了物料主数据标准。通过建立所厂范围内统一的 物料主数据标准, 规范物料主数据的编码、名称及主要信 息的管理规则, 保证数据定义的唯一性、规范性, 方便了 相关人员对数据的统一理解, 有利于跨部门、跨专业进行 数据共享。

\section{3. 数据收集及数据质量提升}

北京航天控制仪器研究所历时 3 个月对重点产品中物 资采购的材料以物料主数据、产品结构树等数据进行收集、 整理、校验。项目组通过下发规范的模板到具体部门, 督 促其限时完成, 并通过数据检查软件以自动篮查重复的数 据。并通过主数据管理软件, 优化设计部门申请新物料编 码流程, 从数据源头避免一物多码。
目前, 完成了 30 余个重点产品结构树、物料主数据、 工艺路线的收集工作，并对 8 万余条物资进行技术性属性 分解、查重、排错等工作。并重点对产品结构树断层或未 分解、型号间数据进行交叉检查、重新给码、数据校验、 完整性分析及评审工作。通过数据审核, 确保这些数据进 入到系统的准确性、规范性和唯一性, 正确指导未来的科 研生产业务以及其他业务。

\section{4. 强化数据全生命周期管理}

相比数据收集的痛苦, 保持数据的“纯洁”更显得重要。 所谓“打天下难, 守天下更难”。在数据从产生到消亡的整 个生命周期中，保持数据的准确性、时效性是一项长期的 工作 $[10]$ 。

航天企业在系统上线运行后, 加强制度建设, 力争做 到以下几点。业务部门的业务流程和操作规程适时调整, 以满足系统内的数据流转要求。系统管理员及时处理系统 中的异常数据, 及时清理垃圾数据和已经失效的数据。另 外, 每周定期对数据进行备份, 确保数据安全, 在出现数 据灾难时可以恢复到最近备份的数据点, 最大限度地减少 损失。

\section{5. 持续推动数据的深度应用}

为了加强数据治理实践, 需要持续推动航天高技术企 业的数据的深度应用 [11]。通过对整合后的业务和产品数 据进行深层次分析, 加强业务自动化、领导驾驶舱、统计 报表、多维比对分析、数据深度挖掘、实时查询等方面的 数据应用。并梳理单位高层决策分析与指挥控制中心大屏 可视化之间的关系, 对业务及产品数据包作深入分析, 挖 掘出数据之间、指标之间的关系, 通过关键指标、跨业务 数据分析等形式, 实现智能决策分析应用 [12], 体现了航 天高技术企业数据治理的成效。

\section{5. 结论}

航天高技术企业通过对主数据管理、数据编码、数据 采集及治理、数据共享、数据利用等方面的持续推动, 将 数据转化成有价值的资产, 还从数据管理组织建设、标准 化体系、质量管理管控、数据全生命周期管理与数据深度 应用等多个方面进行了探索实践。

目前, 航天高技术企业已经吹响了持续开展数据治理 工作的号角, 该项工作的推进必将从根源上提升数据的价 值, 让数据成为新时代的“数字石油”, 不断推动航天高技 术企业向实现航天强国建设战略目标迈进。

\section{参考文献}

[1] 童楠楠,朝乐门.大数据时代下数据管理理念的变革:从结果 派到过程派[J].情报理论与实践,2017,2(40):60-65。

[2] 刘奇燕,张建华.数据治理奠定精益管理基础[J].中国管理信 息化,2015,16(18):86-87。 
[3] 张一鸣.数据治理过程浅析[J].中国信息界,2012,9:15-17。

[4] 苏玉娟. 大数据技术与高新技术企业数据治理创新 [J].科技 进步与对策2016,33(6):47-52。

[5] 凌捷. 大数据时代高新技术企业管理战略转型研究 [J].改革 与战略,2015,5:143-146。

[6] 杨洁. 构建企业级元数据治理体系 [J]. 中国金融电 脑,2012,2:63-65。

[7] 巨克真.魏珍珍. 电力企业级数据治理体系的研究 [J].电力信 息与通信技术,2014(1):7-11。
[8] 陈景文.主数据管理: 打造大数据时代企业核心竞争力 $[\mathrm{J}]$. 福建电脑, 2014,8:75-77。

[9] 张扬.主流主数据管理产品的主数据概念分析 [J].信息系统 工程, 2010,8:33-34。

[10] 王斌,潘新华,谭珂.信息生命周期管理模型在医疗数据保存 中的应用[J].价值工程,2012,31(12):157-158。

[11] 邬贺铨. 大数据时代的机遇与挑战 $[J]$. 求是, $2013,4: 115-118$ 。

[12] 任志锋, 陶立业. 论大数据背景下的政府“循数”治理 [J]. 理论 探索,2014,6:82-86。 